\title{
Synthesis, Growth, Structural, Optical and Electrical Properties of Di-Glycine Sulfate Monohydrate Single Crystals
}

\author{
S. Ramamoorthy ${ }^{* 1}$, M. Angel ${ }^{2}$, M. Manimaran ${ }^{3}$, N.Tamilgandhi ${ }^{1}$, S. Sundaravadivel ${ }^{4}$, \\ R. Anjugam ${ }^{5}$, S.Murugesan 1 \\ ${ }^{1}$ Department of Physics, Vivekanandha Arts and Science College, Tamilnadu, India. \\ ${ }^{2}$ Department of Physics, Vellore Institute of Technology, Vellore, Tamilnadu, India. \\ ${ }^{3}$ Department of Chemistry, Vivekanandha Arts and Science College, Tamilnadu, India. \\ ${ }^{4}$ Department of Physics, Dr. R.K.Shanmugam College of Arts \& Science, Tamilnadu, India. \\ ${ }^{5}$ Department of Physics, Presidency College, Chennai, India.
}

\begin{abstract}
Single crystals of Diglycine Sulphate monohydrate (DGSM) have been grown by slow evaporation solution growth technique. The size of the grown crystals is $20 \times 10 \times 10 \mathrm{~mm}^{3}$ with the duration of 25 days. To identify the cell parameter, space group and planes indexing the grown crystals were subjected to single \& powder XRD. The different modes of vibrations present in the crystal were identified with FT- IR spectrum. The optical transmission, absorbance, extinction coefficient, Reflectance and refractive index have been studied to find its linear properties by UV-Vis spectroscopy. Band gap energy was calculated to be $4.71 \mathrm{eV}$. The dielectric constant, dielectric loss and AC conductivity has been measured as a function of frequency and temperature.
\end{abstract}

KEYWORDS: Solution growth, Single Crystal, Characterization, FT-IR, XRD, UV

\section{INTRODUCTION}

In recent years, complexes of amino acids with organic and inorganic acids possess excellent NLO properties [1-6]. Amino acids are interesting materials for NLO applications, it contains an asymmetric carbon atom so it is optically active and most of them crystallize in noncentrosymmetric space groups. Also, amino acids exist as zwitterionic nature. Glycine is one of the fundamental amino acids and it has the non-chiral zwitterionic form. It crystallizes in six different forms viz. $\alpha, \beta, \gamma, \delta, \varepsilon$ and $\beta^{\prime}-$ forms. $\alpha$-glycine has no asymmetric carbon atom and is optically inactive. It has been reported [7-10] that glycine combines with sulpuric acid, nitric acid, calcium chloride, calcium nitrate, barium chloride, silver nitrate to form useful single crystals. Many of the glycine complexes are observed to be non-centrosymmetric and showing nonlinear optical (NLO) effects widely used application in the area of laser technology, optical communication and data storage technology.

\section{METHODS AND MATERIALS}

Di-Glycine Sulfate Monohydrate single crystal was grown by slow evaporation solution growth technique. Solution was prepared by dissolved analar grade Glycine (Merk) and Sulfuric acid (Merk) in the stoichiometric ratio 3:1 in double distilled water and stirred well for 3 days using a temperature controlled magnetic stirrer to yield a homogeneous mixture of solution. The prepared solution was left to dry and the DGSM salt was obtained. The purity of the synthesized salt was improved by successive recrystallization process and filtration. The size of the crystal was $20 \times 10 \times 10 \mathrm{~mm}^{3}$ and growth period was taken around 25 days. The chosen crystal was already grown in the earlier century [11-13]. The DGSM single crystal was shown in Fig.1.

\section{RESULTS AND DISCUSSION \\ 3.1 Single crystal X-Ray Diffraction Analysis}

The grown crystals were subjected to few characterization namely Single XRD, Powder XRD, UV-Vis Spectroscopy, FT-IR Spectroscopy and Dielectric measurement. In order to reveal the crystal structure, $\mathrm{x}$ ray diffraction studies were carried out and data were shown in Table1. The unit cell parameters obtained. The abc values intersects the axes at hkl and $\alpha \beta \gamma$ are the phase of the sample The crystal system belongs to Monoclinic with P21/c Symmetry [14].

\subsection{Powder X-Ray Diffraction Analysis}

Powder X-ray diffractometer with $(\mathrm{Cu} \mathrm{K} \alpha=1.54056 \AA)$ radiation to study the crystallinity of the grown crystal. This powder pattern is one-dimensional recording of the intensity of diffracted radiation from all the sets of parallel planes as a function of the angle 2 theta within the angular range measured. Finely and crushed powder of the grown DGSM crystalline sample was used for the analysis. Then it was scanned over the range of $10-80^{\circ}$ at a scanning rate of $1 \%$ minute. The intensity of the diffracted beam was recorded as a function of 2 theta and the peaks were indexed [15] and shown in Fig.2

\subsection{Fourier transforms infrared Analysis}

Fourier transform infrared (FT-IR) spectrum was recorded using $\mathrm{KBr}$ pellet technique with a Perkin- Elemer RXI spectrometer. Fig.3 shows the recorded FT-IR spectrum of DGSM crystal in the range $400-4000 \mathrm{~cm}^{-1}$ to identify the functional groups present in the grown crystal [16]. The Table2 was shown the frequency assigned to its relevant groups.

*Corresponding Author: ramjothymani@gmail.com

Received: 15.01.2019 Accepted: 18.02.2019 Published on: 27.02.2019

Ramamoorthy et al., 


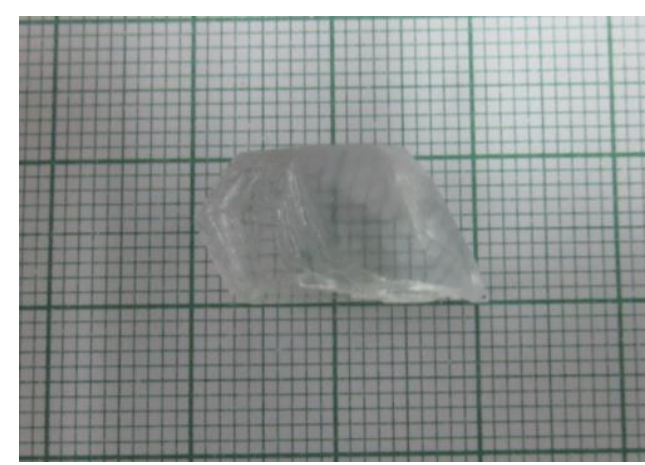

Fig.1 DGSM Single crystals

Table. 1 comparison of crystal data

\begin{tabular}{|c|c|c|}
\hline Parameter & Reported & Present \\
\hline $\mathrm{a}$ & $9.717 \AA$ & $9.73 \AA$ \\
\hline $\mathrm{b}$ & $8.481 \AA$ & $8.50 \AA$ \\
\hline $\mathrm{c}$ & $13.474 \AA$ & $13.51 \AA$ \\
\hline$\alpha$ & $90^{\circ}$ & $90^{\circ}$ \\
\hline$\beta$ & $105.22^{\circ}$ & $105.78^{\circ}$ \\
\hline$\gamma$ & $90^{\circ}$ & $90^{\circ}$ \\
\hline Unit cell Volume & $1071.4 \AA^{3}$ & $1079.5 \AA^{3}$ \\
\hline
\end{tabular}

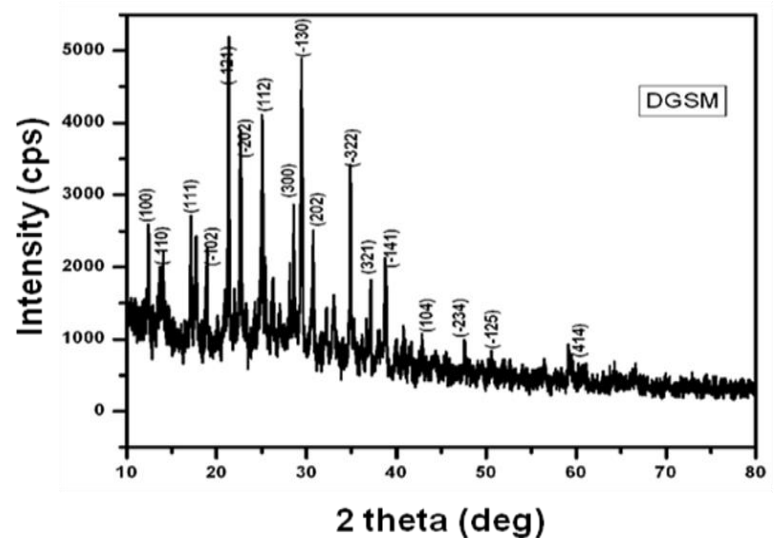

Figure 2 Powder X-Ray Diffraction of DGSM crystal

Table 2. FT-IR Frequency Assignment

\begin{tabular}{|c|c|}
\hline Frequency & Assignments \\
\hline 3549.31 & O-H Weak and broad band \\
\hline 3015.97 & C-H stretching \\
\hline 1732.43 & C=0 carboxyl stretching with broad band \\
\hline 1622.06 & N-H secondary medium sharp band \\
\hline $1473.74,1184.03$ & C-N medium sharp stretching \\
\hline 1276.39 & C-O stretch occurs \\
\hline 1095 & C-N sharp bending \\
\hline 1014.90 & S=O one strong band stretching \\
\hline 852.99 & C-C stretching vibration \\
\hline 460.12 & COO- Rocking \\
\hline
\end{tabular}

Ramamoorthy et al., 


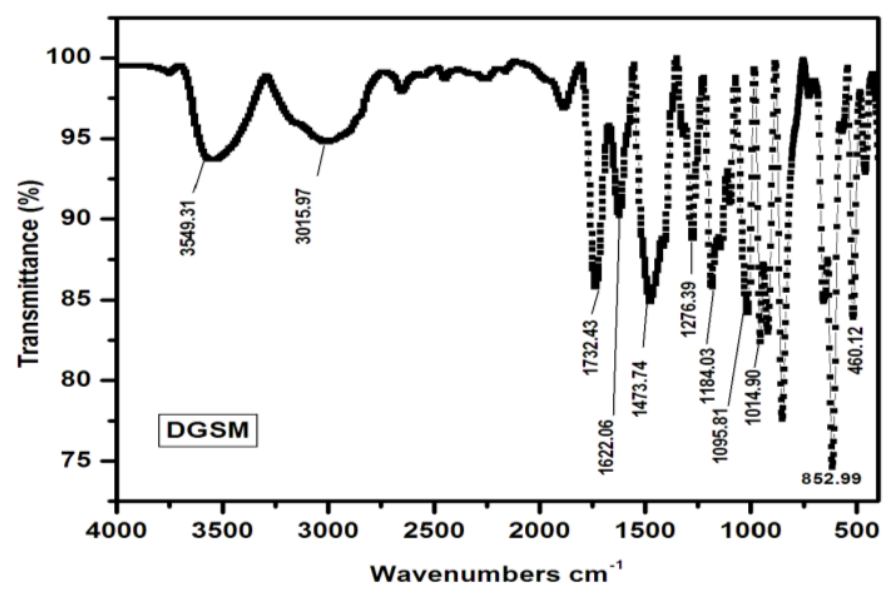

Figure 3 FT-IR spectrum of DGSM single crystals

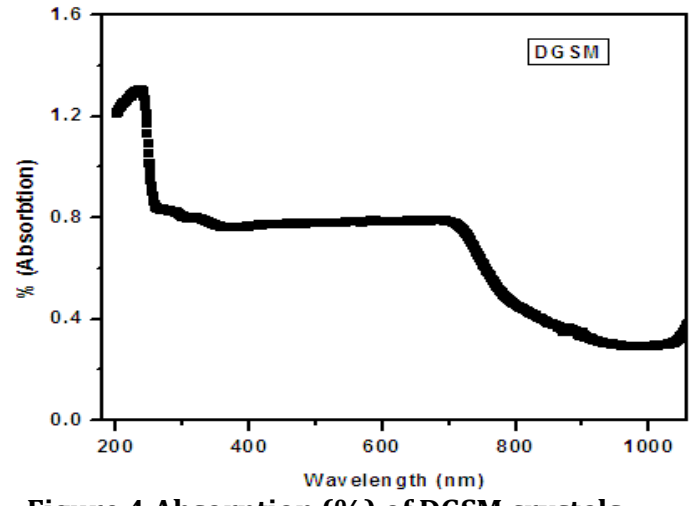

Figure 4 Absorption (\%) of DGSM crystals

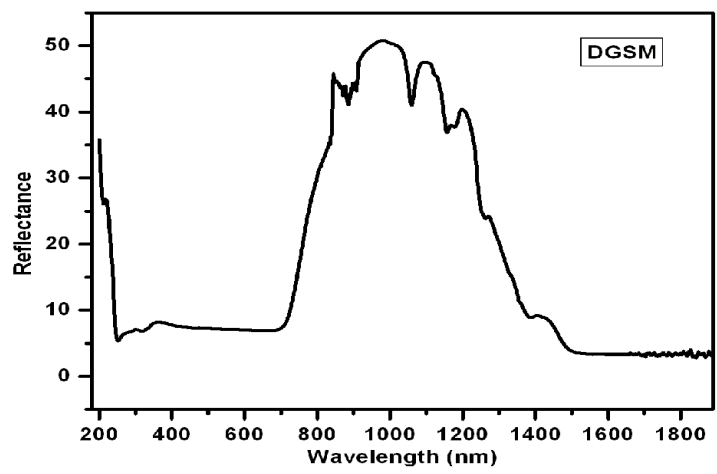

Figure 6 Reflectance of DGSM crystals

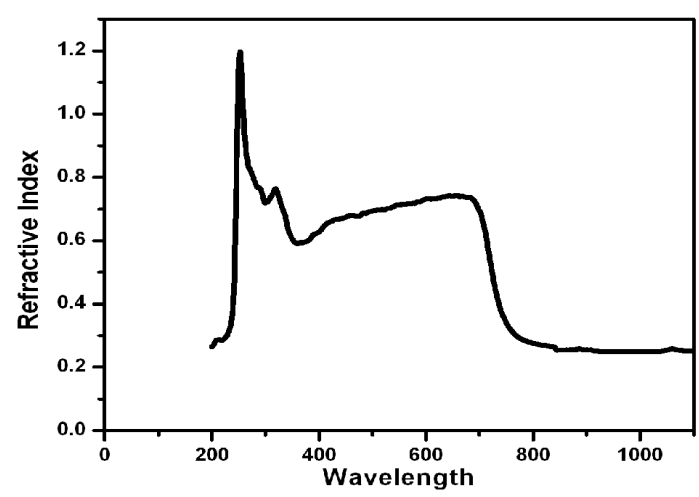

Figure 8 Refractive Index of DGSM crystals

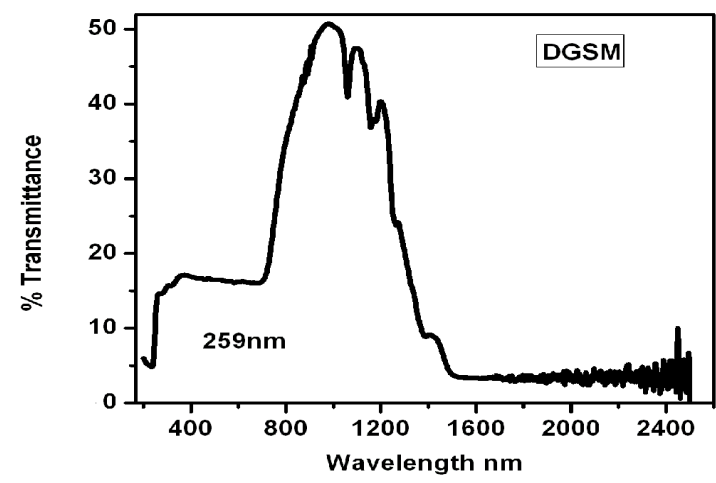

Figure 5 Transmittance (\%) of DGSM crystals

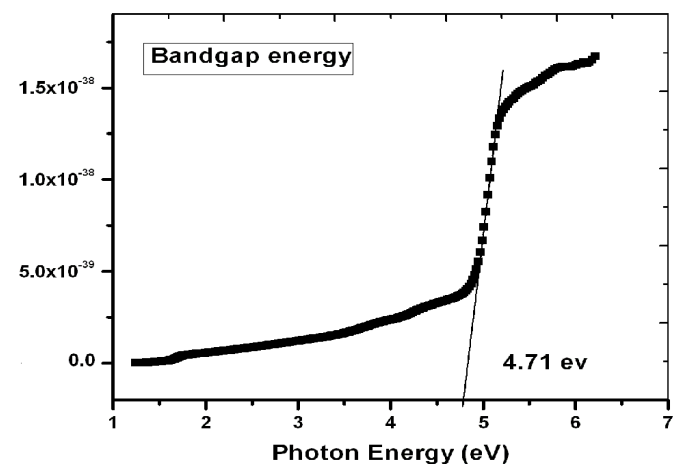

Figure 7 calculated band gap energy of DGSM crystals

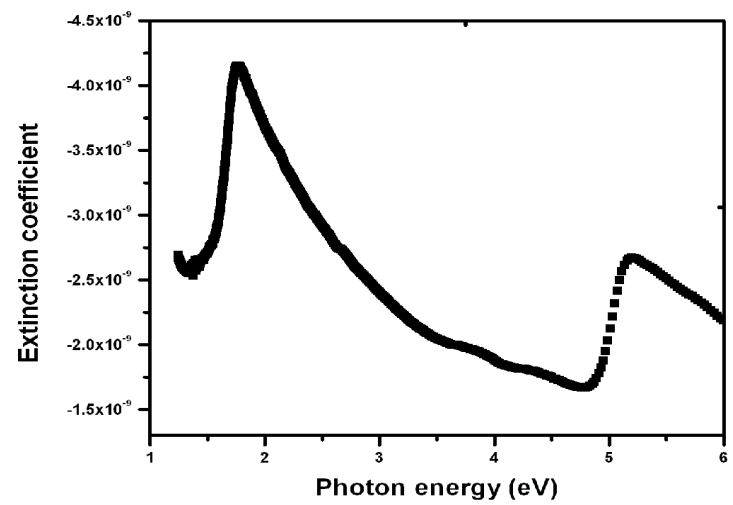

Figure 9 Extinction Coefficient of DGSM crystals

Ramamoorthy et al., 


\subsection{UV-Vis Analysis}

Single crystals are used in optical applications [17-18]. Due to this, optical transparency and UV- cut off are important. UV-Vis transmittance spectrum of DGSM crystal of thickness $2 \mathrm{~mm}$ was recorded using Perkin Elmer-Lambda 35. UV-Vis DRS spectrophotometer in the range of 190- $2600 \mathrm{~nm}$ was used for this study. The room temperature optical properties of the DGSM single crystals was measured and analyzed. The recorded spectrum of absorption (Fig.4), transmittance (Fig.5), reflectance (Fig.6), band gap energy (Fig.7), refractive index(Fig.8) and extinction coefficient (Fig.9) was calculated. It is observed that the Extinction Coefficient (k) increases with increase in wavelength (in terms of photon energy) upto the cut-off wavelength. The low value of is due to the weak interaction between the photons and electrons in the material. The refractive index value was calculated as 1.2 for DGSM.

\subsection{Dielectric Analysis}

The magnitude of dielectric constant depends on the degree of polarization charge displacement in the crystals. The dielectric constant and dielectric loss were measured using Agilent 4284-A LCR meter. The dimensions of the used samples were $2 \times 2 \times 2 \mathrm{~mm}^{3}$. Two opposite surfaces across the breadth of the sample were treated with good quality silver paste in order to obtain good Ohmic contact. Using the LCR meter, the dielectric constant (Fig.10), Dielectric Loss (Fig.11) and AC conductivity (Fig.12) of the crystals were calculated. The dielectric constant of materials is due to the contribution of electronic, ionic, dipolar and space charge polarizations, which depend on the frequencies. At low frequencies, all these polarizations are active. The space charge polarization is generally active at low frequencies and at high temperature. At high frequencies the energy required to rotate the dipole is less since all the polarization mechanisms are not operative therefore, dielectric loss was to minimum and which suggest the less electrical defects and the better quality of the grown crystal. The A.C electric field alters the equilibrium of the phonon system and the subsequent relaxation is associated with energy dissipation [19-21].

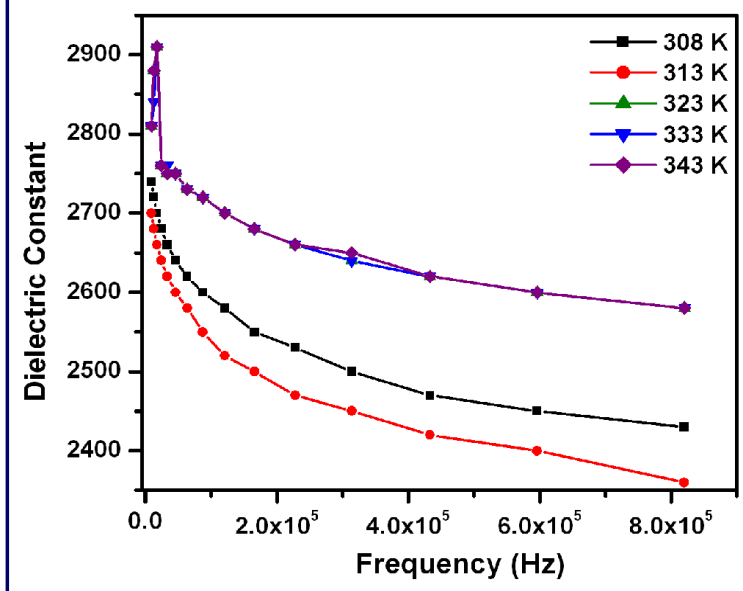

Figure 10 Dielectric Constant

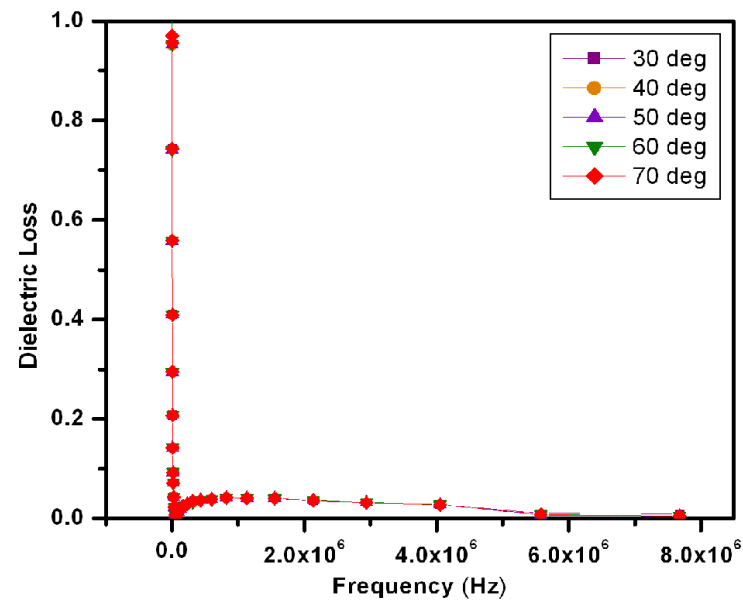

Figure 11 Dielectric Loss

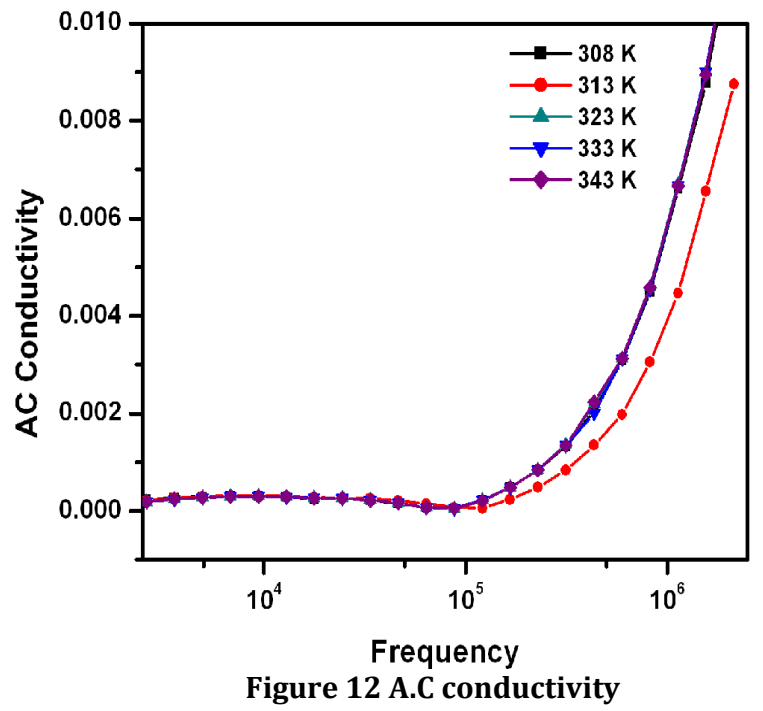

Ramamoorthy et al., 


\section{CONCLUSION}

The lattice dimensions were determined from the single crystal XRD technique and found that DGSM crystal belongs to Monolinic crystal system with noncentro Symmetric space group. The planes are indexed with the help of check cell software in order to find out the morphology of the grown crystals DGSM by powder $\mathrm{X}$-ray diffraction. The presence of functional groups was identified by FT-IR spectral analysis. From the UVVis transmittance study, the band gap energy was found to be $4.71 \mathrm{ev}$. The grown DGSM crystal was belonging to Space charge polarization with help of dielectric studies.

\section{REFERENCES}

[1]. Adhikari, S., Kar, T., 2013. Experimental and theoretical characterization of semiorganic nonlinear optical material L-leucine hydrobromide, Mat. Res. Bull, 48, 1612-1617.

[2]. Xu, D., Wang, X.Q., Yu, W.T., Xu, S.X., Zhang, G.H. 2003. Crystal structure and characterization of a novel organic nonlinear optical crystal: L-arginine trifluoroacetate, J. Cryst. Growth, 253, 481-487.

[3]. Kumar, G.R., Raj, S.G., Mohan, R., Jayavel, R., 2005. Growth and characterization of new nonlinear optical L-threonium acetate single crystals, J. Cryst. Growth, 283, 193-197.

[4]. Natarajan, S., Britto, S.A.M., Ramachandran, E., 2006. Growth, thermal, spectroscopic, and optical studies of L-alaninium maleate, a new organic nonlinear optical material, Cryst. Growth Des, 6, 137-140.

[5]. Devi, T.U., Lawrence, N., Babu, R.R., Ramamurthi, K., 2008. Growth and characterization of Lprolinium picrate single crystal: A promising NLO crystal, J. Cryst. Growth, 310, 116-123.

[6]. Moitra, S., Kar, T., 2008. Second harmonic generation of a new nonlinear optical material Lvaline hydrobromide, J. Cryst. Growth, 310, 45394543.

[7]. Manimekalai, P., Selvarajan, P., 2015. Nucleation kinetics, growth and studies of diglycine magnesium sulfate crystals J. Chem. Pharm. Res, 7, 691-696.

[8]. Esthaku Peter, M., Ramasamy, P., 2016. Structural, growth and characterizations of NLO crystal: Triglycinium calcium nitrate, Adv. Mater. Lett. 7, 1, 83-88.

[9]. Ravi Kumar, S.M., Sivavishnu, D., Sivavishnu, D., Ganapathi, M., VethaPotheher I., Vimalan, M., Chennakrishnan, S., 2016. Synthesis, growth and characterization of (tri) glycine barium chloride single crystal for applications in the domain of optoelectronics and photonics, J Mater Sci: Mater Electron, 27, 10, 10113-10121.
[10]. Sekar, C., Parimaladevi, R., 2009. Effect of silver nitrate (AgNO3) on the growth, optical, spectral, thermal and mechanical properties of $\gamma$-glycine single crystal, Journal of Optoelectronics and Biomedical Materials, 1, 2, 215 - 225.

[11]. Cano, F.H., Martinez-Carrera, S., 1974. The Crystal Structure of Diglyeine Sulphate Monohydrate, Acta Cryst. B, 30, 2729.

[12]. Narayanan, P., Venkataraman, S., 1975. Z.Kristallogra, Crystal strucutre analysis of some addition compounds of glycine, 142, 52-81

[13]. Mohan Rao, J.K., Vishwamitra, M.A., 1972, Crystal structure of glycine silver (I) nitrate Acta Crystallogr., B, 28, 1484.

[14]. Krishnakumar, V., Jayaprakash, J., Boobas, S., 2017. Photoconductivity, dielectric, thermal and mechanical studies on nonlinear optical phasematchable single crystal: 2-amino-4methylpyridinium 4-nitrobenzoate, J Mater Sci: Mater Electron 28, 2, 1706-1714.

[15]. Martin Britto Dhas, S.A., Natarajan, S., 2007. Growth and characterization of a new organic NLO material: Glycine nitrate, Optics Comm. 278, 434-438.

[16]. Iyanar, M., Thomas Joseph Prakash, J., Muthamizhchelvan, C., Ponnusamy, 2009. Synthesis, Growth, and Characterization Studies of a Semiorganic Nonlinear Optical Single Crystal of Gamma Glycine, J. Phys. Sci, 13, 235-244.

[17]. Shanthi, D., Selvarajan, P., Perumal, S., 2016. "Growth, linear optical constants and photoluminescence characteristics of betaalaninium picrate (BAP) crystals" Optik, 127, 6, 3192-3199.

[18]. Vasudevan, V., Ramesh babu, R., Reicher nelcy, A., Bhagavannarayana, G., Ramamurthi, K., 2011. Synthesis, growth, optical, mechanical and electrical properties of L-lysine L-lysinium dichloride nitrate (L-LLDN) single crystal, Bull.Mater. Sci 34, 469-475.

[19]. Rajesh, P., Ramasamy, P., Bhagavannarayana, G., 2009. Effect of ammonium malate on growth rate, crystalline perfection, structural, optical, thermal, mechanical, dielectric and NLO behaviour of ammonium dihydrogen phosphate crystals, J.Cryst.Growth, 311, 4069-4075.

[20]. Vela, T., Selvarajan, P., Freeda, T.H., 2011. Synthesis, growth and material characterization of bis L-alanine triethanol amine (BLATEA) single crystals grown by slow evaporation technique Journal of Minerals \& Materials Characterization \& Engineering, 10, 959-972.

[21]. Theresita Shanthi, N., Selvarajan, P., Mahadevan, C.K., 2009. Growth, structural, mechanical, spectral and dielectric characterization of $\mathrm{NaCl}-$ added Triglycine sulfate single crystals, Current Applied Physics, 9, 1155-1159. 